\title{
Cloud Instrument Powered by Solar Cell Sends Data to Pachube
}

\author{
doi:10.3991/ijoe.v6i4.1464 \\ Silviu Folea ${ }^{1}$, Marius Ghercioiu ${ }^{2}$ and Doru Ursuțiu ${ }^{3}$ \\ ${ }^{1}$ Technical University of Cluj-Napoca, Romania \\ ${ }^{2}$ Cores Electronic LLC, Austin, Texas, USA \\ 3 "Transilvania" University of Brasov, Romania
}

\begin{abstract}
Despite the economic downturn, there have been quite a few new developments in the world of remote measurements lately. Tag4M (www.tag $4 \mathrm{~m} . \mathrm{com}$ ) introduced the concept of cloud instrument where sensors connected to WiFi tags send data to off-the-shelf Access Points which are part of the WiFi infrastructure that exists in enterprises, retail outlets, factories, and warehouses. Access Points route the data to the Internet where specialized web applications receive the information for processing and display. One of these specialized web applications is Pachube, (http://www.pachube.com) which bills itself as a "real-time data brokerage platform". Pachube enables people to tag and share real time sensor data from objects, devices and spaces around the world. This article presents the pachube cloud instrument where sensors connected to Tag4M WiFi tags send digitized data to $\underline{w w w}$.pachube.com for public display. The article contains very detailed analysis of the solar cell power source that is used to continuously power the Tag4M tag during this application. Cloud Instruments powered by solar cells enable people around the world to share real time sensor data using web pages on the Internet. This is a very interesting and exciting technology development that we want to bring to your attention.
\end{abstract}

Index Terms-Tag4M tag, Solar Cell, Wi-Fi Sensors, Web Page Instrument, pachube, green energy.

\section{INTRODUCTION CLOUd INSTRUMENT}

In a new concept known as the Instrumentation Cloud sensors or actuators are connected to the $\mathrm{A} / \mathrm{D}$ or $\mathrm{D} / \mathrm{A}$ front of a sensor tag which sends or receives data and commands to or from a commercially available wireless Access Point (AP) or router. Further, data is routed to an Internet IP address that defines a Server ID. A usercontrolled web-based application server, or Web Page Instrument, receives the data for processing. In the Instrumentation Cloud, the hardware is unchained - measurement front ends are no longer tied to a particular instrumentation network. A Wi-Fi sensor tag connects to any access point or wireless router within range. Even more significant, the software is unchained - measurement data are no longer tied to a particular data acquisition program on a specific computer. Instead it resides in the Internet. That is, instead of a sensor tag sending its data to a device driver that feeds measurement readings into local software on a PC, it sends the data to an AP for further routing to an Internet IP address that defines a Server ID. A user controlled web-based application server, or Web Page Instrument, receives the data for processing. A Web Page Instrument running even on portable devices can provide any number of services: data presentation to an alarming service that responds to dangerous levels by sending a text message or tweet or even a correcting signal to the sensor tag are just a few in a rapidly growing application space [1].

\section{A. www.pachube.com}

In the realm of instrumentation, an interesting precursor of things to come is Pachube, (http://www.pachube.com) which bills itself as a "real-time data brokerage platform". Pachube enables people to tag and share real time sensor data from objects, devices and spaces around the world. You connect sensors to a PC or even a mobile device such as an iPod, and by running applets available on that website, measurement results can be displayed on Pachube so you can examine them at any time from anything that can access the Internet [2].

www.pachube.com is a real time brokerage platform based out of the UK that patches the planet with a blanket of sensors connected to the Internet and the Pachube web page.

\section{B. Extended Environments Markup Language (EEML)}

Extended Environments Markup Language (EEML) is a protocol for sharing sensor data between remote responsive environments, both physical and virtual. It can be used to facilitate direct connections between any two environments; it can also be used to facilitate many-to-many connections as implemented by Pachube. EEML supports installations, buildings, devices and events that collect en-

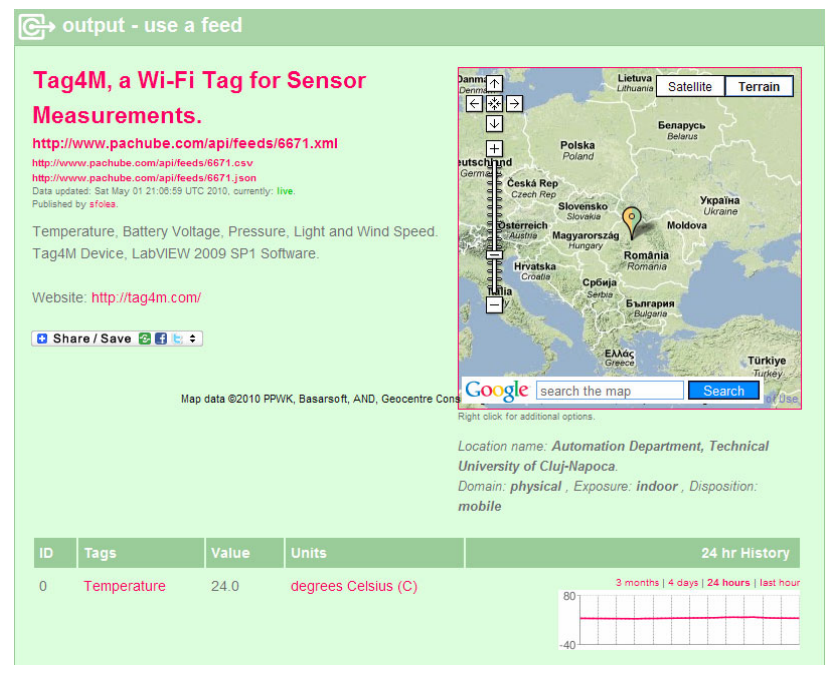

Figure 1. Tag4M Wi-Fi tag sends data to pachube 
vironmental data and enables people to share this resource in real-time either within their own organizations or with the world as a whole via an internet connection or mobile network access. It can enable buildings to "talk", sharing remote environmental sensor data across the network in order to make local decisions based on wider, global perspectives [2].

\section{Green Energy}

There are applications where we want the Tag4M tag to be powered by solar cell in continuous operation mode, but it has to also be feasible, therefore we need to define what feasible means for this application. Solar cell generated power qualifies as green energy, you take the solar energy during the day and spin it in two directions. In one direction you power the tag during the day. The other direction is to store the energy somehow such that at night, when the sun stops shining, you run the tag powered from the stored energy supply. One question of solar cell feasibility is its capability to store day generated energy such that you can use it during the night. Another question of feasibility is the capability of the solar cell generated power to supply enough voltage for tag Wi-Fi communication sessions each wake-up period no matter how many re-transmissions or re-associations with Access Points are needed. Whenever you convert energy from one form to another, the process will have losses. Another aspect of feasibility is to minimize these losses to a level where the size, complexity and cost of solar cell and associated storage and conversion circuitry are sufficiently low to make the solution cost competitive with today's batteries.

\section{Tag4M Tag Powered by Solar Cell}

We have conducted research on the possibility of replacing the Tag4M tag 3V lithium battery with a solar cell based power source. The article gives a detailed description of this research effort and the solar cell power supply for the Tag4M tag that has been designed and prototyped.

The Tag4M tag has a battery holder and it is regularly powered from a $3 \mathrm{~V}$ CR123A Lithium battery.

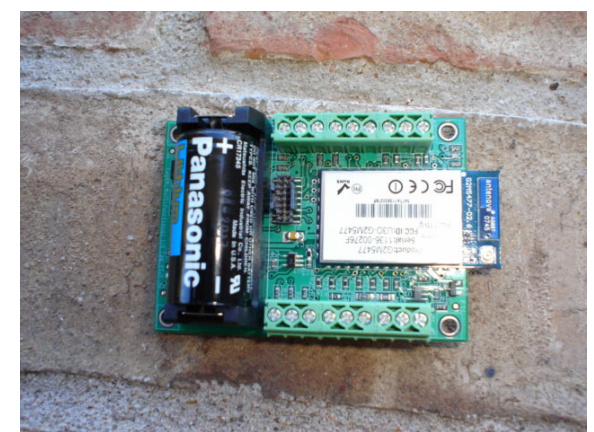

Figure 2. Tag4M tag powered from a 3V Lithium battery

The tag will function on a minimum voltage level of $2.0 \mathrm{~V}$ and up to a maximum voltage level of $3.7 \mathrm{~V}$. Current consumption is the variable that needs to be closely monitored because it is very unevenly distributed over the wake-up period of time. Figure 3 shows tag current consumption levels during one wake-up period of time. You can see the current consumption peaks associated with the following events [3], [4]:

1. boot sequence takes $10-12 \mathrm{~ms}$ and has a mean current consumption of $10 \mathrm{~mA}$;
2. transmission period, where the tag may transmit up to six times inside of a communication period. Transmission time takes 5-10 $\mathrm{ms}$ and has a peak current consumption of $210 \mathrm{~mA}$, which is also the wake-up period pick current consumption level.

3. measurement period takes $0.5-5 \mathrm{~ms}$ and has a mean current consumption of $20 \mathrm{~mA}$;

4. receiving period, when the tag waits for AP acknowledgement, which takes $0 \ldots 80 \mathrm{~ms}$ and has a mean current consumption of $30 \mathrm{~mA}$.

In sleep mode (5) the tag current consumption is $3 \mathrm{uA}$.

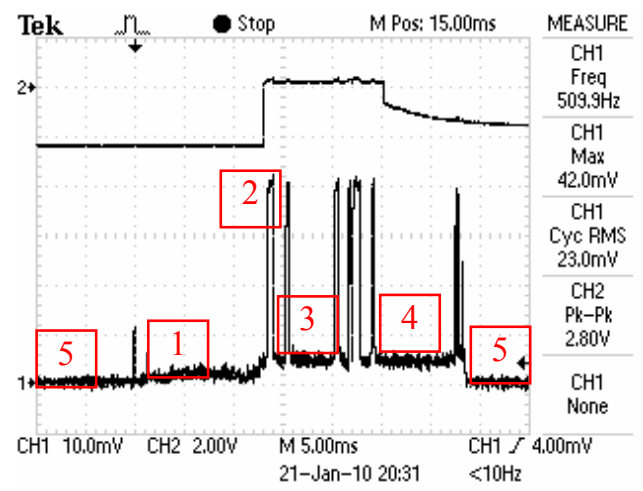

Figure 3. Current Consumption and Measurement Period

Total bi-directional communication time during a wakeup period can be as long as $120 \mathrm{~ms}$, including all possible retransmissions with a minimum of $40 \mathrm{~ms}$ for no retransmissions [5], [6].

One concern when using battery power is the necessity to replace the battery from time-to-time which can be after weeks, months or years of continuous operation depending on power consumption during wake-up time periods and length of the sleep times. The tag can be powered from an external 3.3V DC power adapter. This alternative is always available where feasible and it has no power consumption restrictions. Another alternative is to connect to the external power tag terminals to a power generator assembly that uses "free" energy from natural sources like wind, solar, vibration/movement, water, etc. We will concentrate the discussion in this article on connecting a solar cell to power the Tag4M tag.

\section{A. Solar cell connected directly to tag}

The simplest method to replace the tag battery with a solar cell is to directly connect a solar cell array capable of continuously generating $+2.4 \ldots 3.6 \mathrm{~V}$ and at least $200 \mathrm{~mA}$ directly to tag external power supply terminals Vext (1) and DGND (2).

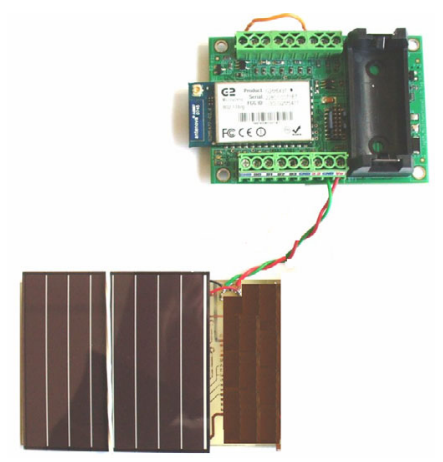

Figure 4. Tag4M tag with solar cell connected directly to tag power terminals 
Different light conditions will influence the level of open circuit voltage and current generated by the solar cell. If the solar cell is not sufficiently illuminated it will not generate enough voltage/current and therefore it will not be capable of powering the tag. Generally speaking, the solar cell will generate a certain amount of power (U*I) for each level of solar illumination intensity. The tag does not need $200 \mathrm{~mA}$ of current continuously; in fact the average current the tag needs during a wake-up period of time is about $100 \mathrm{~mA}$. Problem is that inside this average of $100 \mathrm{~mA}$ the solar cell needs to provide the $210 \mathrm{~mA}$ under a very short period of time needed for transmission. The solar cell that was used in experimentation, AM$8801 \mathrm{CAR}(5.7 \mathrm{~cm} \times 5.5 \mathrm{~cm}, \$ 13.50$ single unit price at Digi-Key) is a medium power cell rated at $50 \ldots 100 \mathrm{~mW}$ that can generate a maximum of $6.8 \mathrm{~V}$ with a short circuit output current of $20 \mathrm{~mA}$. The more current the tag needs the lover the available voltage, for $210 \mathrm{~mA}$ needed during Wi-Fi transmission the available solar cell voltage output is about $500 \mathrm{mV}$. Clearly, this solar cell is not capable of powering the tag if directly connected [7].

\section{B. Solar Cell connected to capacitor array}

A minimal improvement to this setup is the addition of a few capacitors to collect the solar energy. The solar cell, when illuminated, generates voltage that charges the capacitors which act as a buffer of energy storage. The tag needs to be continuously powered by voltage in the range 2 to $3.7 \mathrm{~V}$. In sleep mode the tag consumes current in the range 3 to $10 \mathrm{uA}$. Every wake-up period of time the tag needs an average of $100 \mathrm{~mA}$ of current except for very short instances when it needs $210 \mathrm{~mA}$. A higher solar cell output voltage across the capacitor will provide a faster charge-up time for different lighting conditions. If the solar cell has a higher output voltage, then we do not need to worry about enough current being supplied, but rather we need to build a stabilizing circuitry to cap the generated voltage at $3.7 \mathrm{~V}$. If a lower current solar cell is used, like the AM-8801CAR (20.4mA), then we need to set a sleep time that is sufficiently long to give the solar cell time to charge the capacitor array with enough power to carry the next wake-up period. Experiments show that this solar cell connected to the capacitor array is capable of powering the tag continuously under perfect illumination conditions if sleep time is set to a minimum of 1 second and wake-up periods are no longer than $120 \mathrm{~ms}$. This is to say that the solar cell generates $20 \mathrm{~mA}$ continuously, which charges the capacitor array buffer and the tag uses $100 \mathrm{~mA} 10 \%$ of the time.

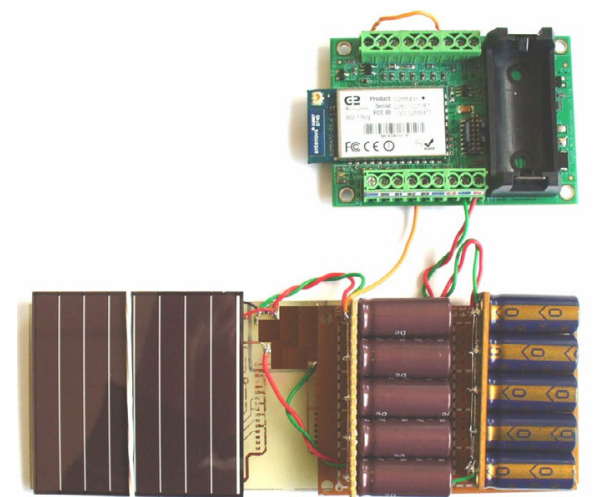

Figure 5. Tag4M tag with solar cell connected to capacitor array
Poor lightning conditions will downgrade the level of open circuit voltage and current generated by the solar cell in this setup. Generally speaking, the tag operating mode being a succession of wake-up and sleep time periods is very well suited to the idea of harnessing power from the environment and also to the idea of an energy buffer. It would be perfectly feasible to continuously power the tag with the solar cell array connected to the capacitor array, if enough solar energy is harnessed from the environment to supply the tag while in wireless transmit mode. Our experiment shows that a solar cell with medium power rated at only $50 \ldots 100 \mathrm{~mW}$ if coupled to a $10 \times 3.3 \mathrm{mF}$ capacitor array will generate enough power for the tag but only under very good illumination conditions. This excludes night time and clouded days.

\section{Linear voltage regulator circuitry}

Further improvement of this solution can be achieved by using a more powerful solar cell connected to the capacitor array via a linear voltage regulator circuitry containing a low-dropout (LDO) voltage regulator to limit the voltage drop to only the saturation voltage which is $3.3 \mathrm{~V}$. During sleep time periods, when the tag uses only 3 to $10 \mathrm{uA}$ or current from the energy buffer, solar cell generated voltage could rise above the maximum allowed of $3.7 \mathrm{~V}$ and this voltage level may damage the tag circuitry. The linear voltage regulator circuitry will act like a shield by stabilizing solar cell output voltage to $+3.3 \mathrm{~V}$ which is what the tag needs.

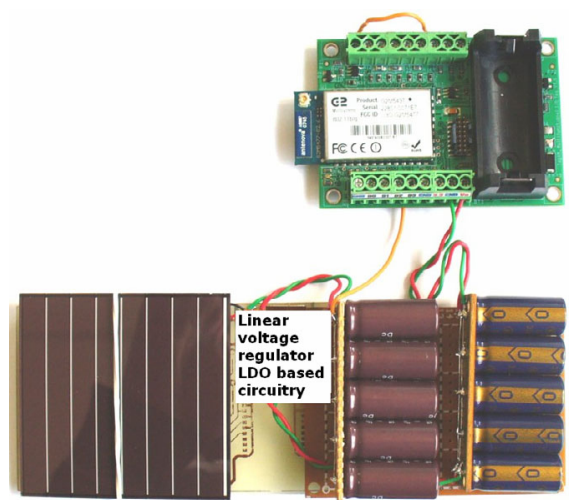

Figure 6. Tag4M tag with solar cell connected to capacitor array via a linear voltage regulator

The linear voltage regulator circuitry is simple to implement and very low cost but has considerable losses because the source itself uses part of the generated power. Results from experimentation using the AM-8801CAR solar cell indicate that if the solar cell is located inside an office building with indirect light then tag sleep time setting has to be in the 10 to 25 seconds range at a minimum. The linear regulator circuitry solves the problem of the solar cell being able to supply enough voltage to cover wake-up period voltage usage including Wi-Fi transmissions under very good illumination conditions that need to be correlated with the length of sleep time periods.

This solution does not provide enough voltage for tag operation during the night and nor for the situation where the tag does not associate immediately with an AP and it has to retry several times or it has to re-transmit several consecutive times because of loss of data. Also, the LDO needs higher input voltage, 0.1 to $0.3 \mathrm{~V}$ on top of the generated output voltage. This requirement translates in hav- 
ing to select a solar cell that is capable of generating at least $3.6 \mathrm{~V}$.

\section{Burst power regulator}

Further improvement of the solar cell conditioning circuitry requires a high efficiency, fixed frequency, BuckBoost DC/DC converter like the LTC3440 (\$5.83 single unit price Digi-Key) to provide high efficiency, low noise, burst power for the tag. This circuitry is a replacement of the linear voltage regulator circuitry. Burst power means almost no power during tag sleep time periods and highly efficient output voltage that is equal to the input during tag Wi-Fi transmission sessions inside wake-up periods. The part uses only $25 \mathrm{uA}$. The DC/DC converter part has an efficiency of 65 to $95 \%$ when generating the $3.3 \mathrm{~V}$ necessary for tag operation from an input voltage as low as $1.8 \mathrm{~V}$. The part uses as little as $25 \mathrm{uA}$. Compare that with an LDO current consumption of 0.3 to $1 \mathrm{~mA}$ which represents almost $10 \%$ of what the solar cell can generate [8].

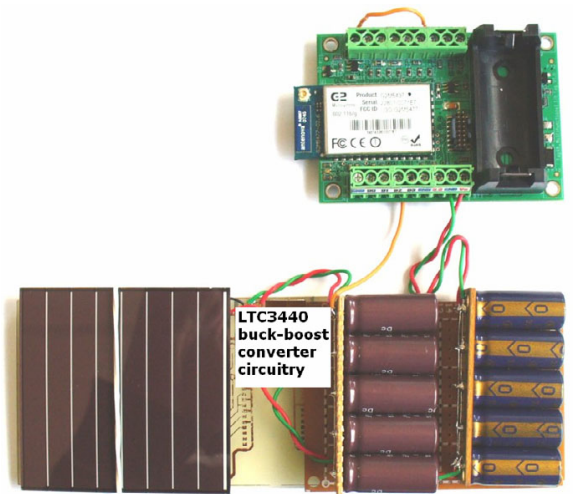

Figure 7. Tag4M tag with solar cell connected to capacitor array via a buck-boost converter circuitry

By using the buck-boost converter circuitry we increase solar cell efficiency. This solution applied to a solar cell of type AM-8801CAR and the same capacitor array (2 sets of 5 capacitors of $3.3 \mathrm{mF}$ each) as in the previous experiments supports continuous tag functionality with sleep time of 1 second and receive time of $200 \mathrm{~ms}$ which is very close to maximum tag functional capacity. If the solar cell is placed in the shadow or solar illumination is less powerful at times the immediate consequence on tag functionality will be to make sleep time longer in the $10,25,60$ s range depending on generated voltage value. The dependency between voltage output, sleep time period and illumination conditions leads us to the idea that we may want to read the voltage that is generated by the solar cell using a tag voltage input tag channel - in order to set the appropriate sleep time period.

\section{E. Accumulator with charger}

Further improvement of the solar cell conditioning circuitry would be the complete solution which is a high efficiency circuitry that insures tag power under any conditions of illumination - poor illumination, shadow, inside building, night, etc - and also any number of retransmissions and associations with Access Points. This can be implemented by using an accumulator (energy storage device) of type Li-Ion or Ni-Mh and a charger circuitry like the LTC1734-4.1 (\$3.33 single unit price Digi-Key).

The accumulator offers a larger storage capability than the capacitor array. In effect the accumulator is equivalent with a very high - 3.3 to $70 \mathrm{~F}$ type $\mathrm{HZ} / \mathrm{HW}$ - capacitor arrays. The solar cell charges the accumulator during the day while also powering the tag. If the accumulator is small, like the one used in this setup $3.7 \mathrm{~V} / 200 \mathrm{mAh}$ then we will keep half of the capacitor array. If the accumulator is big enough we do not need the capacitor array. We can also eliminate the accumulator and use higher capacitor arrays.

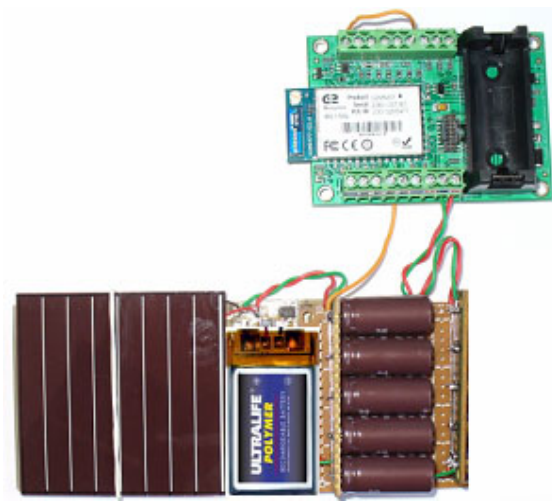

Figure 8. Tag4M tag with solar cell connected to capacitor array via a buck-boost converter circuitry, Li-Ion Accumulator and charger

Either way, the most efficient tag operating mode is to continuously read the voltage generated by the solar cell and set the appropriate sleep time period. Make this task part of each tag wake-up period to insure continuous power availability to the tag.

\section{Cloud Instrument Powered By SOlar Cell SENDS DATA TO PACHUBE}

We are using a Tag4M tag powered from a solar cell connected to capacitor array via a buck-boost converter circuitry with Li-Ion Accumulator and charger. The tag performs remote posting of sensor measurement data into the Pachube web site (http://www.pachube.com).

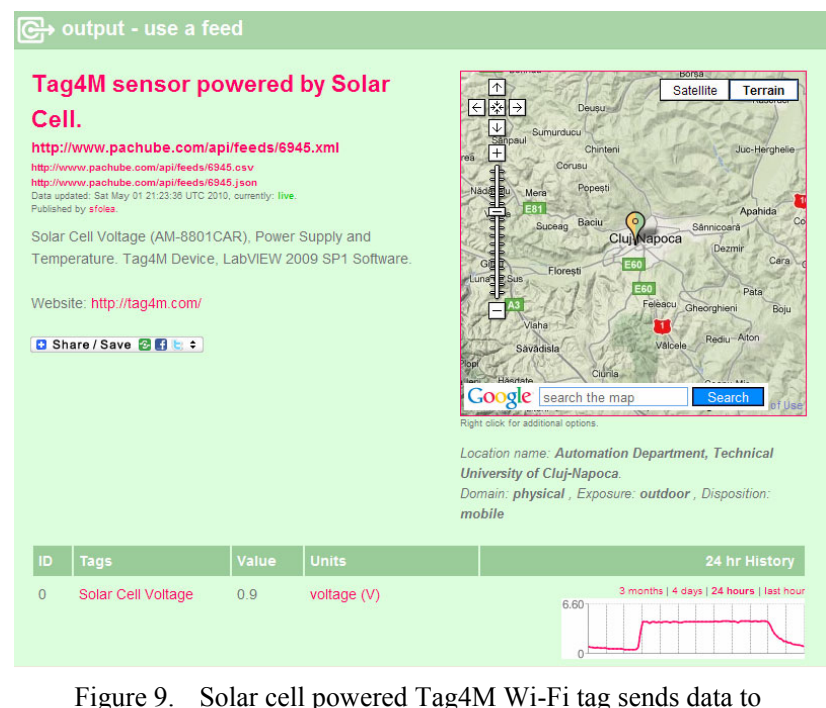

Figure 9. Solar cell powered Tag4M Wi-Fi tag sends data to http://www.pachube.com/feeds/6945

In this setup the sensors connected to the Tag4M tag are located in the Automation Department of the Technical University of Cluj-Napoca, Romania. Data from the tag goes to a LabVIEW application running in a local computer that sends it as an EEML script via TCP/IP over the Internet to the Pachube site in the UK where it is made 
available from any browser at http://www.pachube.com/feeds $/ 7277$.

The following is a more detailed description of this cloud instrument.

In order to send sensor data to Pachube you need to bundle it into an EEML script that Pachube understands. The Tag4M-to-Pachube Cloud Instrument has the following elements:

- Sensors connected to Tag4M tags

- Application running on a local PC that is connected to the Internet. Application reads sensor data from Tag4M tag via Wi-Fi, bundles the data into an EEML script that Pachube understands and sends it via TCP/IP Write commands to the Internet and www.pachube.com

- Pachube web site captures the data and posts it on the Internet in a feed that is associated with a number for unique identification (ex: http://www.pachube.com/feeds/7277). Pachube sends acknowledgement messages to the local PC running the local application in order to let the user know that the data has been received and it is posted.

\section{A. Sensors connected to the Tag4M tag}

In this particular application the tag monitors the following values [9]:

- Solar cell generated voltage (the AM-8801CAR solar cell output voltage line is connected to the $0-10 \mathrm{~V}$ line of the tag).

- Internal tag power supply voltage.

- Temperature sensor (thermistor) located on the tag.

- Sleep time settings.

- Temperature sensor TC1046VNB connected to tag line AI2 [10].

- Pressure sensor MP3H6115A6U connected to tag line AI1 [11].

- Humidity sensor HIH-5030/5031 connected to tag line AI0 [12].

\section{B. Application running on a local PC}

The application running on the local PC feeds data to Pachube. This application may be written in any language, there is no special requirement other than that the $\mathrm{PC}$ is connected to the Internet and the application runs continuously and feeds data to the Pachube web site. Pachube allows for two feed methods from the local application:

- Feed data when asked by Pachube (on demand), or

- Feed data periodically at predefined (by the Pachube client) time intervals

We chose to write the local application in LabVIEW. The main VI named Pachube Update Feed 6945.vi uses feed method b). Pachube Update Feed 6945.vi implements the following steps:

- Read sensor data from the Tag4M tag. Tag channel $0-10 \mathrm{~V}$ is connected to the solar cell and measures the generated voltage. If solar cell generated voltage is lower than $4.2 \mathrm{~V}$ then change sleep time to $120 \mathrm{sec}$ onds. If generated voltage is higher than $4.2 \mathrm{~V}$ then sleep time is set to 25 seconds. This is an example of sleep time control based on solar cell generated voltage value.

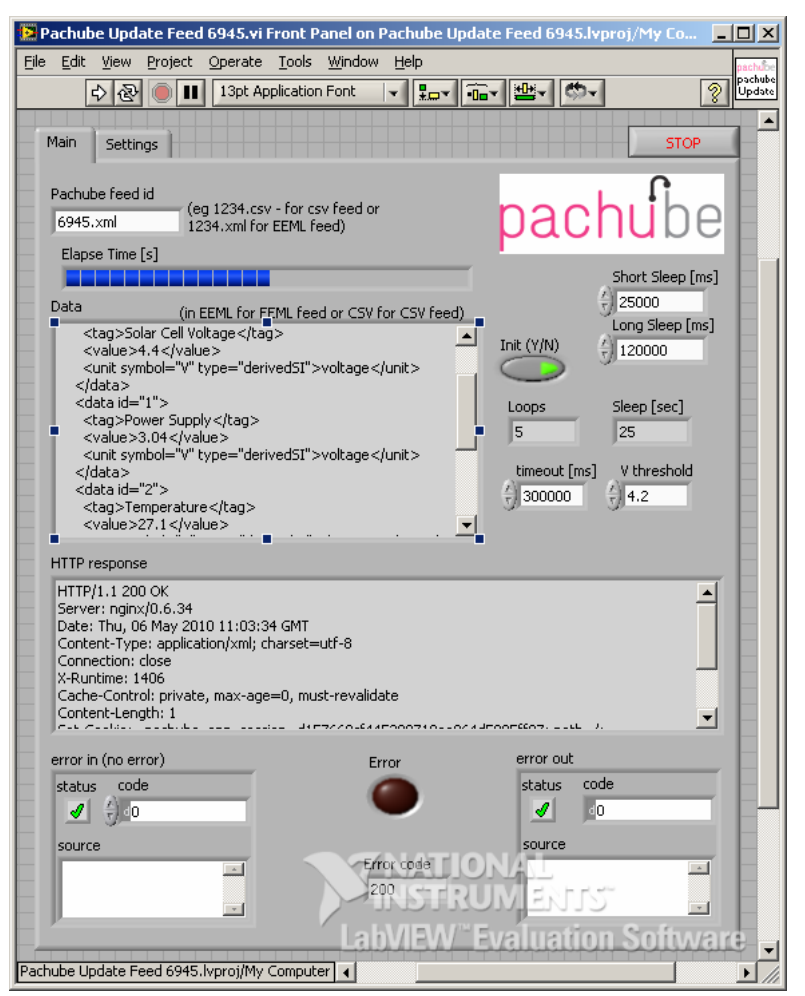

Figure 10. Tag4M an Pachube VI application panel

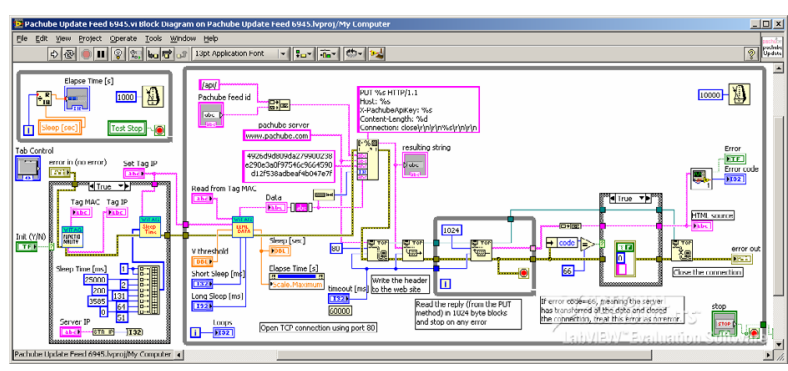

Figure 11. Tag4M an Pachube VI application panel

- Package the data into an EEML script format that is understood by the Pachube web site.

- Open a TCP/IP connection between the computer running this application and the Internet. TCP/IP Write the EEML script to www.pachube.com and read reply in 1024 byte blocks from Pachube. If data has been received by Pachube, close the TCP/IP connection and start allover again by reading the tag.

\section{Pachube web site captures the data and posts it for} public display

Pachube "talks" EEML which is a protocol for sharing sensor data between remote responsive environments. The local application packages data from sensors into the following standard EEML script:

$<$ ?xml version="1.0" encoding="UTF-8"?>

$<$ eeml xmlns="http://www.eeml.org/xsd/005">

$<$ environment>

$<$ data id="0" $>$

$<$ tag $>$ Solar Cell Voltage $</$ tag $>$

$<$ value $>$

$</$ value $>$

$<$ unit symbol="V" type="derivedSI" $>$ voltage $</$ unit $>$

$</$ data $>$ 


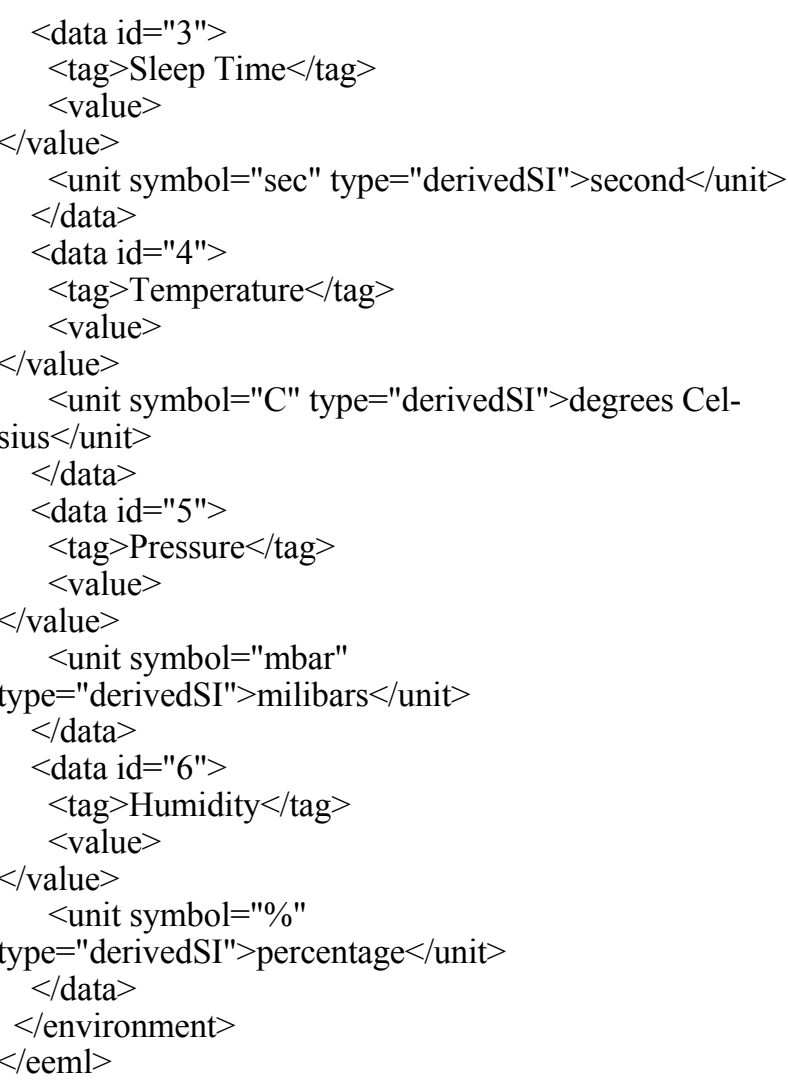

The EEML script is sent by the local computer via TCP/IP and port 80 to www.pachube.com. When this data arrives to Pachube the web site will post a feed containing information about its location and sensor data that has been posted in the script.

\section{DOOR OPEN FOR INNOVATION}

This merging of the latest hardware technologies RFID and Wi-Fi tags powered by solar cell energy send data to Access Points - and software technologies - web page instruments posted on the Internet like www.pachube.com and http://demo.tag4m.com/ - now allows engineers to find all sorts of innovative applications that require sensor measurements.

Tag4M works with innovators of sensor technology to create web page instruments and widgets for the PC, iPhone and other portable Wi-Fi platforms. We are very excited to have demonstrated in this article that the Tag4M tag can be powered using solar cell generated energy in a very reliable and low cost solution which involves energy storage and application software control of the sleep time period. We hope this is the opening of a new chapter in sensor technology and imaginative uses will emerge for the solar cell powered Instrumentation Cloud in the near future.

\section{REFERENCES}

[1] Tag4M, "Tag4M - The Multifunctional I/O Wi-Fi Tag", Technical Report. Copyright 2007-2010, Austin, Texas, USA, tag4m.com

[2] Pachube, "Store, share \& discover realtime sensor ...", API Documentation, 2010.

[3] G2 Microsystems, "G2C547 SoC Data Sheet", product brief, G2 Microsystems, Inc., Campbell, CA, USA, 2008.

[4] G2 Microsystems, "G2C547 Software, Example Application", Technical Report. G2 Microsystems, Inc., Campbell, CA, USA, 2008.

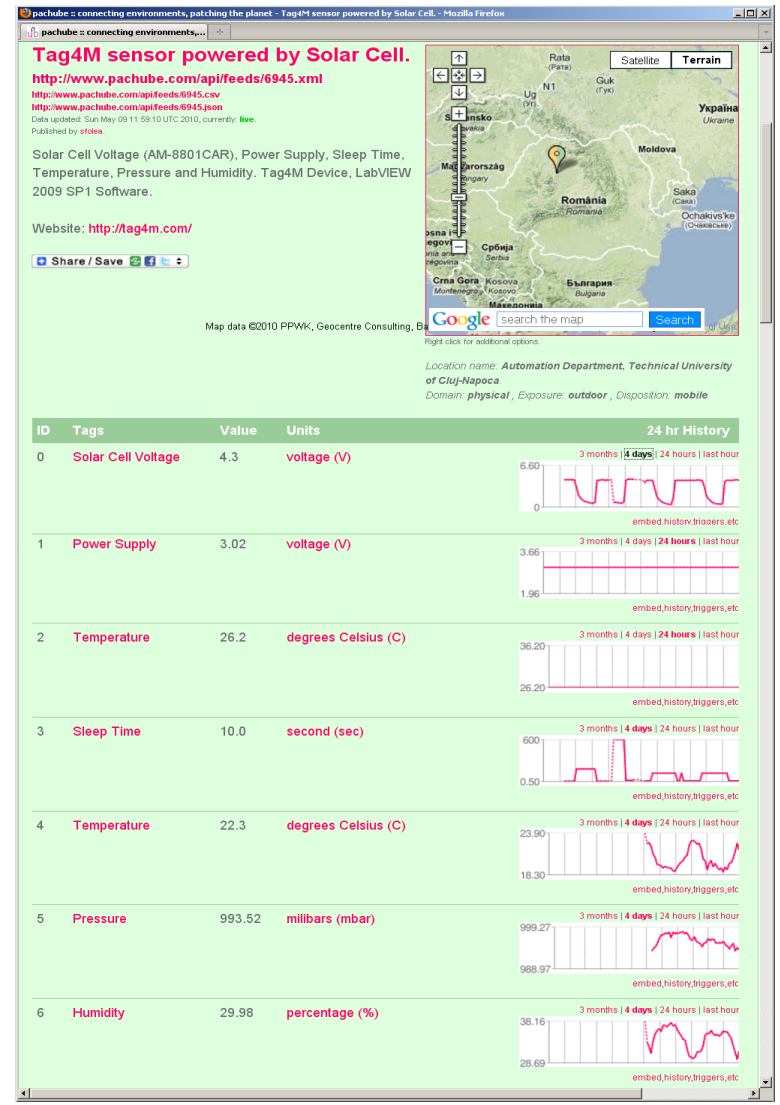

Figure 12. Tag4M VI, data on Pachube at http://www.pachube.com/feeds/7277

[5] G2 Microsystems, "G2M5477 Wi-Fi Module Data Sheet", Technical Report. G2 Microsystems, Inc., Campbell, CA, USA, 2009.

[6] G2 Microsystems, "Data Sheet G2C547 and G2M5477", Technical Report. G2 Microsystems, Inc., Campbell, CA, USA, 2009.

[7] Sanyo, "Amorphous Solar Cell", Datasheet, AM-8801CAR, Tokyo, Japan.

[8] Linear Technology, "Micropower Synchronous Buck-Boost DC/DC Converter", Datasheet, LT 0507 REV B, USA.

[9] S. Folea, M. Ghercioiu, "Tag4M, a Wi-Fi RFID Active Tag Optimized for Sensor Measurements", InTech Education and Publishing, Austria, 2009, ISBN 978-953-7619-72-5.

[10] Microchip, "High Precision Temperature-to-Voltage Converter", Datasheet, DS21496B, USA.

[11] Freescale Semiconductor, "High Temperature Accuracy Integrated Silicon Pressure Sensor for Measuring Absolute Pressure, OnChip Signal Conditioned, Temperature Compensated and Calibrated", MP3H6115A, Rev 4, 10/2009.

[12] Honeywell, "HIH 5030/5031 Series, Low Voltage Humidity Sensors", 009050-2-EN IL50 GLO, 2010, USA.

\section{AUTHORS}

S. Folea is with the Automation Department from Technical University of Cluj-Napoca, Cluj-Napoca, 400027, Romania (e-mail: silviu.folea@aut.utcluj.ro).

M. Ghercioiu is president of Cores Electronic LLC, Austin, Texas USA (e-mail: info@tag4m.com). Tag4M is a trademark of Cores Electronic LLC.

D. Ursutiu is with the University "Transylvania" from Brasov - Romania and manages the Creativity Laboratory, (e-mail: udoru@unitbv.ro).

Submitted May $10^{\text {th }} 2010$. Published as resubmitted by the authors October $17^{\text {th }}, 2010$. 